Journal Section

\title{
IOData: A Python Library for Reading, Writing, and Converting Computational Chemistry File Formats and Generating Input Files
}

\author{
Toon Verstraelen $^{1^{*}}$ | William Adams ${ }^{2}$ | Leila Pujal ${ }^{3}$ | \\ Alireza Tehrani $^{3}$ | Braden D. Kelly ${ }^{2}$ | Luis Macaya ${ }^{5}$ | \\ Fanwang Meng ${ }^{2}$ | Michael Richer ${ }^{2}$ | Raymundo \\ Hernández-Esparza ${ }^{2}$ | Xiaotian Derrick Yang ${ }^{2,4}$ | \\ Matthew Chan ${ }^{2}$ | Taewon David Kim² | Maarten \\ Cools-Ceuppens $^{1}$ | Valerii Chuiko ${ }^{2,6}$ | Esteban \\ Vöhringer-Martinez $^{5^{*}}$ | Paul W. Ayers ${ }^{2^{*}}$ | Farnaz \\ Heidar-Zadeh ${ }^{1,3 *}$
}

${ }^{1}$ Center for Molecular Modeling (CMM),

Ghent University,

Technologiepark-Zwijnaarde 46, B-9052,

Zwijnaarde, Belgium

${ }^{2}$ Department of Chemistry and Chemical Biology, McMaster University, Hamilton, Ontario, L8S-4L8, Canada

${ }^{3}$ Department of Chemistry, Queen's University, Kingston, Ontario, K7L-3N6, Canada

${ }^{4}$ Laboratoire de Chimie Théorique, Sorbonne Université, Paris, 75052, France

${ }^{5}$ Departamento de Físico Química, Facultad de Ciencias Químicas, Universidad de Concepción, 4070371 Concepción, Chile

${ }^{6}$ Faculty of Physics; Taras Shevchenko National University of Kyiv, 01601 Kyiv, Ukraine

IOData is a free and open-source Python library for parsing, storing, and converting various file formats commonly used by quantum chemistry, molecular dynamics, and plane-wave density-functional-theory software programs. In addition, IOData supports a flexible framework for generating input files for various software packages. While designed and released for stand-alone use, its original purpose was to facilitate the interoperability of various modules in the HORTON and ChemTools software packages with external (third-party) molecular quantum chemistry and solid-state density-functionaltheory packages. IOData is designed to be easy to use, maintain, and extend; this is why we wrote IOData in Python and adopted many principles of modern software development, including comprehensive documentation, extensive 
testing, continuous integration/delivery protocols, and package management. This article is the official release note of the IOData library.

\section{KEYWORDS}

quantum chemistry, molecular mechanics, computational chemistry, file format conversion, data parsing, basis set conversion, input file generation, JSON schema, chemistry software development, theoretical chemistry Python library

\section{1 | WHAT IS IODATA?}

IOData is a free and open-source Python 3 library for reading and writing formatted (non-binary) files generated by a broad array of molecular quantum chemistry, molecular dynamics, and plane-wave density-functional-theory programs. IOData is primarily intended as a utility for (a) parsing molecular/periodic geometry, wavefunction, and trajectory data from various file formats, (b) storing this data in a user-friendly object, so that it can be used in external Python modules, (c) dumping geometry, wavefunction, and trajectory data in a variety of standard file formats, hence (d) converting one standard file format to another, and (e) generating input files for various computational chemistry software packages. Section 5 details the file formats and input file generation currently supported by IOData; however, we designed the IOData framework to be not only easy to use but also easy to extend to new data formats and software.

\section{2 | HISTORY}

During the course of our scientific research, and especially our development of the HORTON[1] and ChemTools[2] software packages, we needed a utility that allowed us to parse the output files of various electronic structure theory programs and dump data from our software packages using standard file formats. Some of this functionality existed in HORTON 2.x [1], but in the process of writing HORTON 3, we decided to split IOData (as well as much of the other functionality of HORTON) into stand-alone libraries to better support modularity, maintenance, and ease of use.

\section{3 | ABOUT IODATA}

IOData is, and always will be, a free and open-source library distributed under the GNU General Public License. The IOData source code is maintained on the GitHub platform; see https://github.com/theochem/iodata, and its documentation is hosted on Read the Docs; see https://iodata.readthedocs.io/en/latest/index.html. We strive to ensure that the IOData source code and website itself is comprehensively documented, including useful tests, scripts, and examples. As that documentation is maintained with the software, providing detailed (and eventually outdated) release notes here seems unwise. Instead, we will briefly list the distinguishing features and key capabilities of IOData in section 5 and demonstrate them in section 6 . 


\section{4 | WHY IODATA?}

Before starting to work on IOData, we considered using other free and open-source utilities for parsing and converting file formats like OpenBabel[3] (mostly geared toward chemoinformatics), RDKit [4] (limited wavefunction capabilities), cclib[5] (limited capabilities for dumping output files), ASE[6] (limited features for molecular systems), MultiWFN[7] (difficult-to-extend Fortran 90 source code), MDTraj [8] and MDAnalysis [9, 10] (both geared towards trajectory formats and analysis). However, none of these achieved our criteria of being easy to use and modify, readable, stand-alone, modular, and written in Python. Most critically, most other software packages are limited in their ability to parse molecular wavefunction information and extremely limited in their ability to write popular quantum chemistry output file formats.

Anticipating that other researchers may have similar needs that can be met by directly using IOData or by building on top of it, we decided to release IOData as a free and open-source library. Nonetheless, we wish to acknowledge the utility of these other programs, which we also use in our research. OpenBabel[3] is extremely useful for cheminformatics tasks; RDKit is an excellent tool for generating molecular structures and conformations; cclib has refined capabilities for parsing an abundance of information from quantum chemistry output/log file formats; ASE supports an abundance of solid-state electronic structure programs; MDTraj and MDAnalysis support an impressive number of trajectory formats; MultiWFN has comprehensive post-processing capabilities and supports many of the most important formats supported by IOData, but does not produce an internal data structure (e.g., a dictionary or a class) that is easily used in other programs. While IOData overlaps with these tools in some respects, its emphasis on reading and writing molecular quantum chemistry data file formats, generating input files, and usability as a library within other programs is unique.

In our view, the distinguishing characteristics of IOData are its modularity, its flexibility, and its ease of use. We achieve this by designing a unifying framework in which data from various file formats can be loaded, stored in a versatile container class, and dumped in a desired file format. We provide comprehensive documentation and rigorously adhere to high coding standards, including comprehensive testing of code correctness, quality, and readability. While IOData is primarily intended to be used as a Python library and its API was designed to facilitate interoperability with other Python packages, many operations can be performed through its command line tools.

\section{5 | FEATURES OF IODATA}

We display various features of IOData by first discussing file formats that it can currently analyze. Specifically,

- The file formats (and their commonly used extensions) that can be both loaded and dumped by IOData include: basic XYZ Cartesian coordinate files ( $\left.{ }^{*} . x y z\right)$, extended XYZ files containing additional atomic properties ( ${ }^{*}$.extxyz), Gaussian[11] formatted checkpoint files (*.fchk), wavefunction files ( ${ }^{*}$.wfn), extended wavefunction files ( ${ }^{*}$.wfx), MultiWFN files ( ${ }^{*}$.mwfn), Molden[12] files (*.molden), Molekel files (*.mkl), Mol2 files (*.mol2), Molpro[13] 2012 FCIDUMP integral files (*.molpro), spatial data files ( ${ }^{*}$. sdf), protein data bank files (*.pdb), QCSchema[14] files ( ${ }^{*}$.json), and cube files (*.cube). Most often, these file formats each contain information on a single chemical compound, and are commonly used in exchanging information between various software packages (e.g. for postprocessing). Several of these file formats are often used to store information on multiple compounds in a single file (e.g. concatenation of a collection of molecules/frames in a single file with a single format). In this regard, IOData also supports load and dump functionality for concatenated * .xyz, *.extxyz, *.fchk, *.mol2, *.sdf, and *.pdb file formats. 


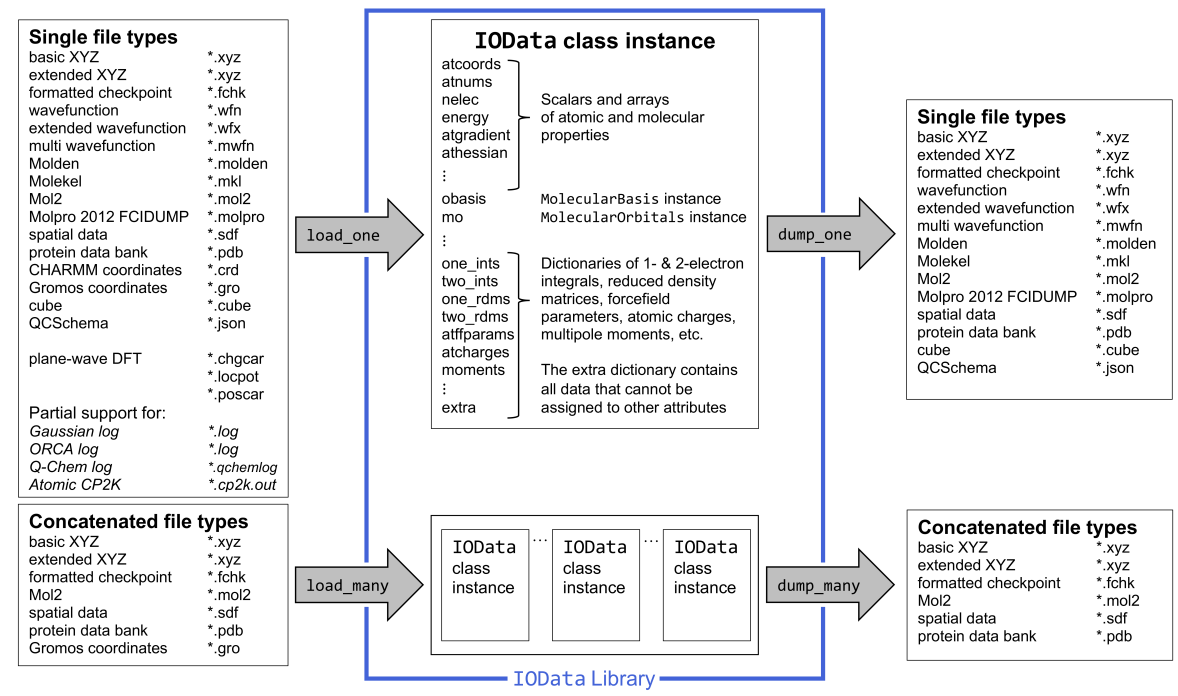

FIGURE 1 Schematic representation of IOData library.

- The file formats (and their commonly used extension) that can be only loaded by IOData include certain files from plane-wave density-functional theory codes including VASP (*.chgcar, *.locpot, *.poscar), molecular dynamics packages like Gromacs (*.gro) and CHARMM (*.crd) coordinate files, and GAMESS[15] punch files (*.dat). IOData can also partially parse and store data from log files of several quantum chemistry software packages including Gaussian[11] log files (*.log), ORCA[16] log files (*.out), Q-Chem[17] log files ( ${ }^{*}$.qchemlog), and atomic CP2K[18] log files (*.cp2k.out). If the user is interested in a specific information printed in these log file that is not currently loaded by IOData, the existing parser can be easily extended.

- To support a broad range of external software, IOData supports, and interconverts between, arbitrary basis-set conventions, including basis-function order, sign (of the spherical harmonics), and normalization ( $\mathrm{L}^{1}$-normalized, $\mathrm{L}^{2}$-normalized, and unnormalized). IOData also supports basis set conversion (e.g. Cartesian to/from spherical, primitive to/from contracted).

- IOData can be used to generate input files for various quantum chemistry and molecular dynamics software packages, including Gaussian[11] and ORCA[16]. Users have the option of providing an input template for specific use cases or using pre-defined basic templates. The input file writer can be easily extended for use with other software packages.

Considering the large number of file formats commonly used in computational chemistry, the IOData library is particularly useful as it provides a user-friendly platform to load the information contained in various file formats into an easy-to-use object and to convert one file format to the other. IOData's file-conversion capability is especially useful because software packages usually work with specific file formats. Obviously, the XYZ and cube file formats cannot be used to generate file formats containing wavefunction information, however, any of the above mentioned formats can be loaded by IOData (like fchk) and converted to any other format (like XYZ, wfn, molden, etc.).

As depicted in Figure 1, the IOData library stores the loaded data as attributes of the IOData class. These are mostly numerical data, arrays, or strings; if available, the information related to molecular basis set and orbitals are 
stored as the 'obasis' and 'mo' attributes, which are instances of the MolecularBasis class and Molecular0rbitals class, respectively. Occasionally, file formats include information on 1- and 2-electron integrals, reduced density matrices, multipole moments, atomic charges, and/or force field parameters; these are each stored as dictionaries. Any other additional information that does not fit into a pre-existing field is stored in the 'extra' dictionary attribute of the IOData class; items in 'extra' may become standard attributes in future releases.

We aim to ensure that these input files can be parsed robustly, so that even when popular software packages fail to follow the technical specifications of a file format (usually because the file format was not strictly or publicly defined) the error is automatically diagnosed and corrected if possible. For example, many programs read and generate *.wfn files that do not follow the original format statements from Richard Bader's AIMPAC program, and no formal file format specifications for ${ }^{*}$.wfn seems ever to have been published. To remedy this, we looked at four ${ }^{*}$.wfn formats from various open-source projects and wrote $a^{*}$. wfn format specification that was as flexible as possible, yet still supported all four versions. The file format specification is provided as part of the IoData documentation, and hopefully will prevent rogue * ${ }^{*}$ wfn file formats in the future. Another example is that different programs (PSI4[19], ORCA[16], Turbomole[20], Molden[12], Molekel, Q-Chem[17], etc.) generate ${ }^{*}$.molden files with different basis-set normalization, sign, and ordering conventions. We automatically detect these conventions by evaluating the atomicorbital overlap matrix, and then store the basis set in a flexible and universal way. When writing a *.molden file, we always use the same normalization convention that the Molden program itself uses.

Among the recently proposed file formats, IOData supports loading and dumping QCSchema[14], a programagnostic JSON schema that serves as both an input and output file format. Unfortunately, the implementation of the QCSchema in MolSSI projects such as QCElemental[21] has diverged from the original QCSchema specification[14]. The version implemented in QCElemental has transformed from a single, extended schema to four different subschema components: Molecule, Input, Output, and Basis. This reinvention of QCSchema is reasonable, but only exists as a Python-based partial implementation in QCElemental, not a true JSON schema specification usable by other potential adopters. IOData implements a backward-compatible and complete version of QCSchema by adopting many of the changes found in QCElemental and the development branch of QCSchema while also providing a genuine JSON schema and extensive documentation specifying each of the four sub-schema.

IOData uses an extensive automatic-testing framework to verify that the code behaves correctly, both when introducing code changes and when making new installations on various operating systems. Currently, IOData uses pytest framework and the unit test coverage feature of codecov . io to ensure a significant portion of our code is well tested; At present, $96 \%$ of the code base is tested. In addition, IOData uses an extensive set of quality-assurance tools (pylint, autopep8, pycodestyle, pydocstyle, etc.) to ensure that the code quality is not degraded by new contributions and the code remains readable and well-documented. The testing and quality-assurance workflows are automatically run by our continuous integration system every time a code change is proposed to our GitHub repository. Currently, we use travis.io for continuous integration, delivery, and deployment. If travis.io reports that the unit tests or quality-assurance tools is failed, the proposed changes are automatically rejected. When our code is ready for release, we Git tag a commit with a special keyword and travis. io builds and tests a release with the Conda and Pip package managers. This allows users to simply install IOData and all its binary dependencies in one command. Developers can also publish new releases to users in one command thanks to the automation provided by this workflow. 


\section{6 | EXAMPLES}

For the most updated documentation and examples on how to use IOData, please refer to the IOData website. Here, we showcase several ways IOData can be used and incorporated into various workflows. Please note that these examples are based on version 1.0 of IOData, and the user might need to modify them if using future major releases of the IOData library. Within minor and bug-fix releases, backward compatibility is guaranteed.

\section{1 | Using IOData as a Python library}

Loading File Formats: The primary use case for IOData is to parse files of various formats and make the loaded data easily accessible to the user. This is mainly facilitated through the load_one and load_many functions, which return an IOData instance and a list of IOData instances, respectively, in which the loaded data is stored. For example,

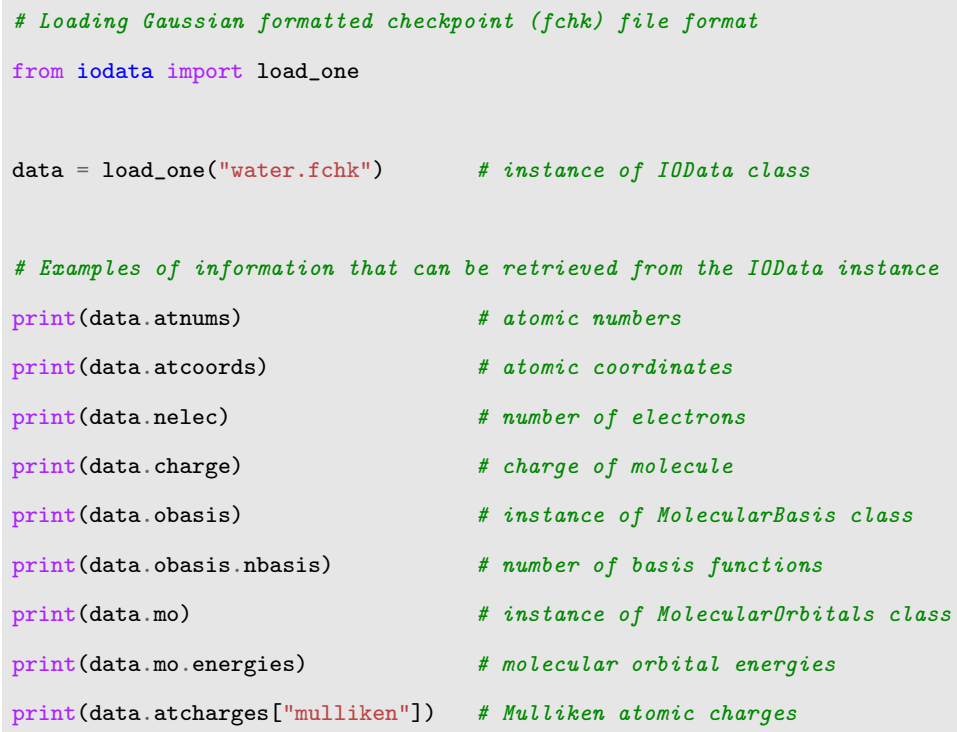

The format of the file specified on line 4 is derived from the file extension, however, the user can override the default file format detected using the optional fmt argument. The above example displays only a few IoData attributes; the data available from each file format is clearly tabulated on the IOData website. It is important to note that the wavefunction information is stored in an IOData instance in a manner designed to simplify calculations. For example, one can simply compute the spin-summed density matrix, for both restricted and unrestricted Slater determinants, using,

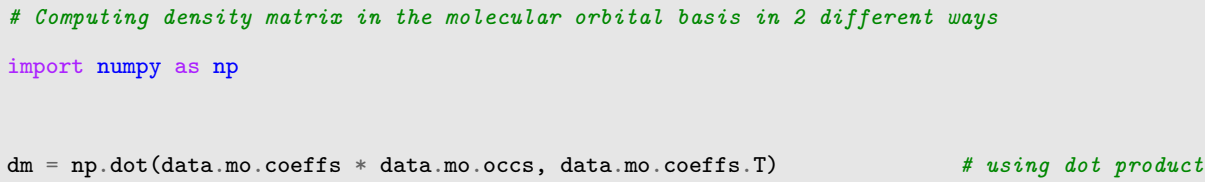


Similarly, the load_many function can be used to load concatenated file formats like databases $\left({ }^{*}\right.$. sdf, ${ }^{*}$. mol $2,{ }^{*} . x y z$, or *.extxyz) or successive conformations of molecules from molecular dynamics trajectories or reaction paths (*.pdb, ${ }^{*}$.fchk, *.xyz, or *.extxyz), stored in a single file. For example,

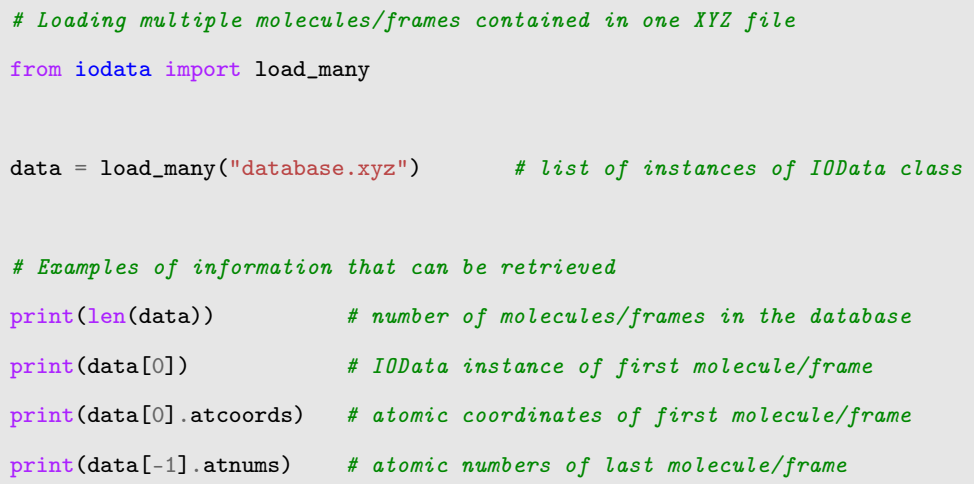

Because many file formats are not strictly defined, the IOData library must be extremely flexible. For example, for the $X Y Z$ file format, IOData accepts atomic numbers, atomic symbols, or mixtures of both as atom specifications in the first column of the Coordinates section. IOData also allows the user to provide their own format specifications. For example, the many versions of the extended $X Y Z$ file format store various atomic properties by appending columns to the basic $X Y Z$ format. IOData can be instructed to load this additional data through the optional atom_columns argument of the load_one or load_many functions.

Writing File Formats: The information loaded from one file format can be used to write output in a different file format, if the required data is available. This is made possible through the dump_one and dump_many functions, which take an IOData instance and sequence of IOData instances, respectively, and write out the specified file format. For example,

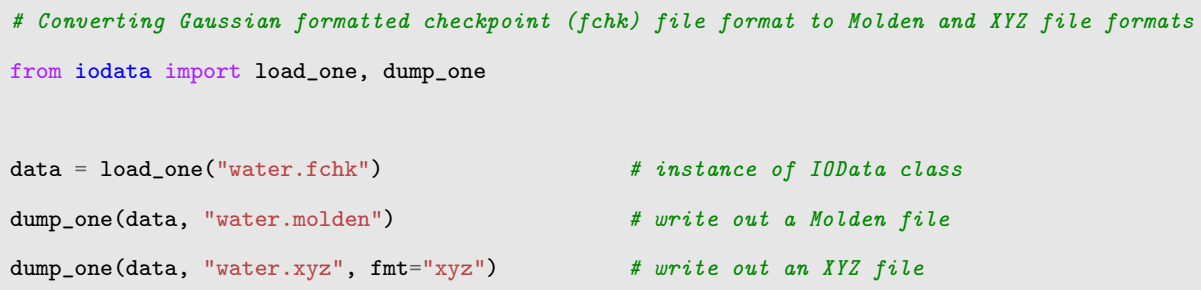

As mentioned previously, the format of a dumped file is derived from the filename extension; however, the user can override this default by specifying the optional fmt argument. Similarly, having a sequence of IOData instances, the dump_many function can be used to generate a single file containing the molecules/frames in the user-specified file format. Similarly, to generate an extended $X Y Z$ file, additional columns can be specified through the optional atom_columns argument of the dump_one or dump_many functions. 
Storing Data: An instance of the IOData class can be constructed by the user, for example, in the process of performing a quantum chemistry calculation. This is helpful for both using the stored information internally (e.g. when using the HORTON 3 and ChemTools[2] software packages) or writing it out in a specific file format. For example,

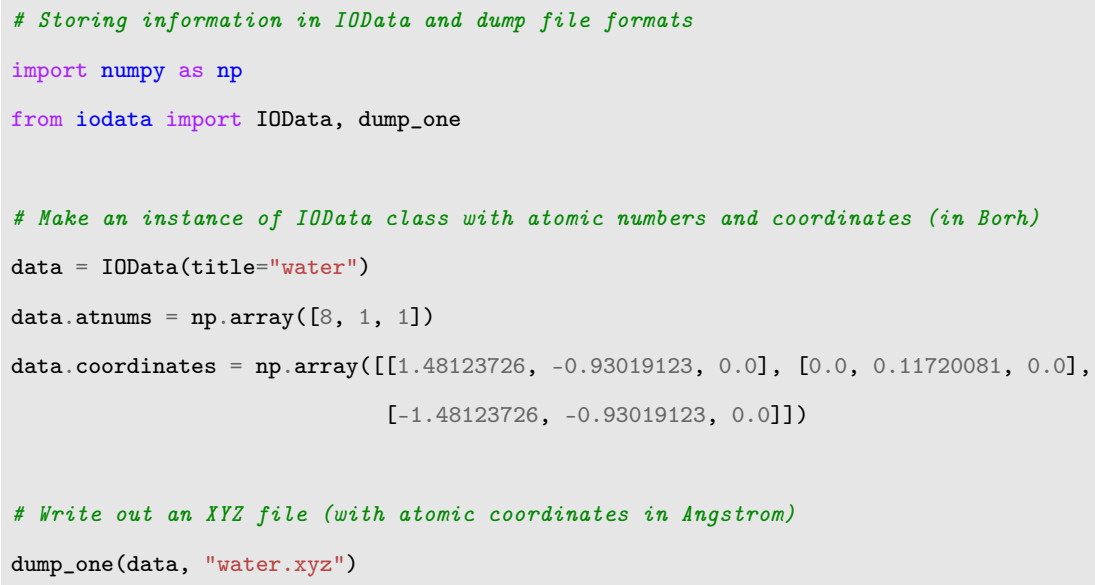

Writing Input Files: Input files for common software packages can be generated using either built-in or user-defined templates. The basic input writing functionality requires only an IOData instance with sufficient data for the input file (i.e. level of theory, basis set name, etc.). For example,

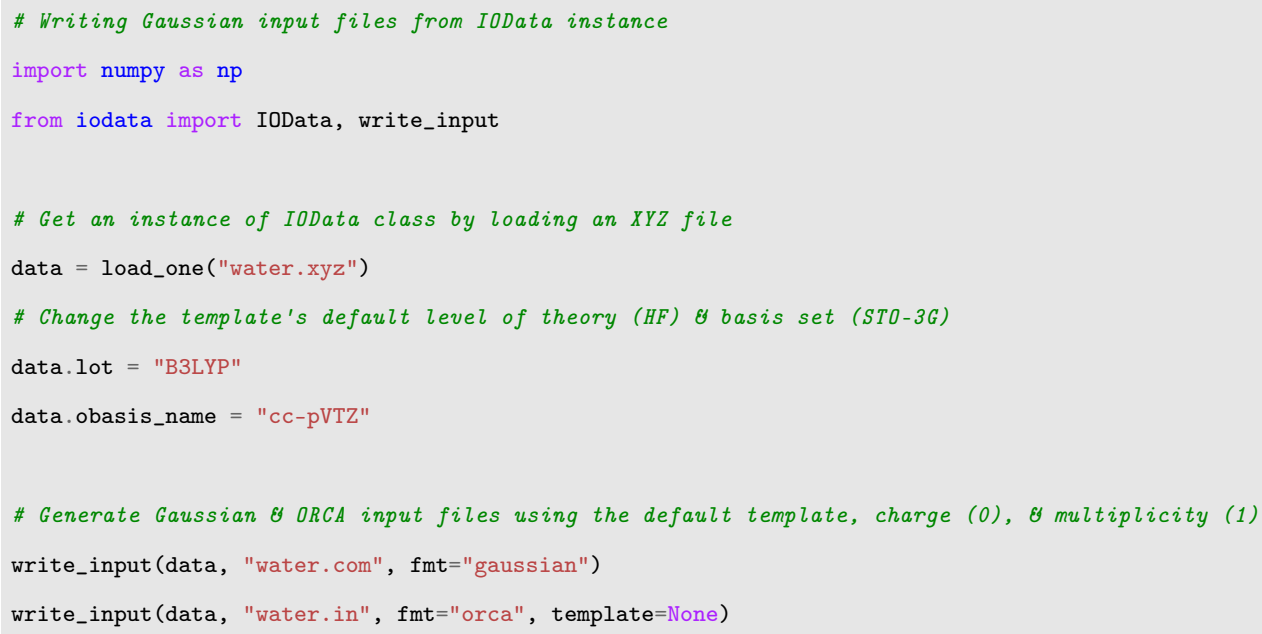

The write_input function accepts a user-defined input template through template argument which is a text file containing Python string substitution markers and IOData attribute names, like $\$\{$ lot $\}$ for level of theory, $\$\{$ obasis_name\} for basis set name, etc.. 


\section{2 | Using IOData as a command-line tool}

iodata-convert is the command-line file-convesion interface accessible upon installing the IoData library. For example,

The input and output file formats are derived from the file extensions, however, they can be specified through optional command-line arguments. To obtain more information on how to customize this utility, please refer to its help message,

\section{7 | FREQUENTLY ASKED QUESTIONS}

Who is IOData for? We intend IOData to be primarily used by computational chemists and researchers developing quantum chemistry software, especially post-processing and visualization tools. Our goal is for IOData to be easy to use for novice programmers and computational chemists, yet capable of supporting advanced quantum chemistry workflows. Achieving these goals requires adherence to software design best practices, so extending or contributing to IOData requires intermediate-level programming ability (i.e. knowledge of decorators, type hints, automatic testing, GitHub, continuous integration and automatic release, etc.). The command-line tools in IOData can be used by anyone who has passing familiarity with Linux shell commands.

What is the mission of IOData? We wish to serve the community of quantum chemistry users and developers by removing the barrier imposed by the plethora of (sometimes obscurely defined and/or documented) file formats. This is clearly a Sisyphean task, but we sincerely hope that others in the community will join our efforts and, if possible, eventually agree on a universal file format.

What does IOData do? As elaborated in sections 5 and 6, IOData currently parses various file formats from thirdparty software packages and makes the data available to Python programs or writes out the data into a different format, and thus supports file format conversion. The latter can also be achieved through command-line functionality of IOData. In addition, IOData generates input files for various quantum chemistry or molecular dynamics software packages using basic or user-defined input file templates.

What is the future direction of IOData? We aim to support parsing and writing additional file formats, and also to extend our support for writing input files and enable direct integration with QCEngine[21]. Our current to-do list for IOData can be found on its GitHub's issue page, and we anticipate that most items will be resolved by the end of 2020. Within our software development efforts, the IOData will eventually serve as the input/output module of the HORTON3 and ChemTools[2] software packages. 
How do I install IOData? The IOData library can be installed from its source code available on GitHub or through pip and conda package-management systems. Maintaining the source code on GitHub allows users to access our latest development, even before it is officially released. For the most updated instructions on how to install IOData, please refer to the IOData website.

Can I contribute to IOData? Yes! We welcome and support new contributions in accordance with our Code of Conduct and Contributing Guidelines. For the most up-to-date instructions on how to contribute, please refer to the IOData website.

\section{8 | SUMMARY}

The purpose of this brief paper is to present the IOData module of HORTON 3, which parses, stores, and dumps a variety of file formats. The most unique capabilities of IOData are its robustness (e.g., its ability to read *.molden files with nonstandard basis-set conventions; its ability to read *.wfn files with strange fixed-formatting issues; the ability to read QCSchema with incompatible formats), its utilities to read and parse files for single and multiple structures (e.g. trajectories, reaction pathways, potential energy surface scans), its scope (including file formats typically used in Gaussian-basis-set-based molecular chemistry software, plane-wave density-functional-theory codes, and molecular dynamics calculations), its ability to output a variety of useful file formats, its meticulous documentation and testing protocols, its ease-of-use and extensibility, and its command-line tools. While we will continually improve IOData, we believe its present functionality already has significant benefits to the broader community of theoretical and computational chemists and welcome their comments and contributions.

\section{9 | RESEARCH DATA STATEMENT}

IOData is free and open-source software; the examples used herein (or embellishments thereof) are disseminated with the package, which is available on GitHub; https://github.com/theochem/iodata.

\section{Acknowledgements}

We wish to acknowledge various refinements to the IOData library from Steven Vandenbrande, Jennifer Garner, Thomas Pigeon, Stijn Fias, Ali Malek, and the HORTON development team. T.V. acknowledges the Foundation of Scientific Research-Flanders (FWO) and the Research Board of Ghent University (BOF) for their financial support. L.M.Z. and E.V.M. acknowledge financial support by CONICYT/FONDECYT/REGULAR/FOLIO 1200369, PCI CONICYT INSTITUTO MAX PLANCK FOR TERRESTRIAL MICROBIOLOGY MARBURG MPG190003 and the Max-Planck Society. P.W.A. acknowledges Natural Sciences and Engineering Research Council (NSERC) of Canada, the Canada Research Chairs, Compute Canada, and CANARIE for financial and computational support. F.H.Z. acknowledges financial support from FWO, NSERC and Queen's University Research Initiation Grant.

\section{Conflict of Interest}

There is no conflict of interest. 


\section{references}

[1] Verstraelen T, Tecmer P, Heidar-Zadeh F, González-Espinoza CE, Chan M, Kim TD, et al., HORTON 2.1.1; 2017. https: //theochem.github.com/horton/.

[2] Heidar-Zadeh F, Richer M, Fias S, Miranda-Quintana RA, Chan M, Franco-Perez M, et al. An explicit approach to conceptual density functional theory descriptors of arbitrary order. Chemical Physics Letters 2016;660:307-312.

[3] O'Boyle NM, Banck M, James CA, Morley C, Vandermeersch T, Hutchison GR. Open Babel: An open chemical toolbox. Journal of Cheminformatics 2011;3(1):33.

[4] Landrum G, RDKit: Open-source cheminformatics;. https://www.rdkit.org/.

[5] O'boyle NM, Tenderholt AL, Langner KM. cclib: A library for package-independent computational chemistry algorithms. Journal of Computational Chemistry 2008;29(5):839-845. https://onlinelibrary.wiley.com/doi/abs/10.1002/jcc.20823.

[6] Larsen AH, Mortensen JJ, Blomqvist J, Castelli IE, Christensen R, Dułak M, et al. The atomic simulation environment-a Python library for working with atoms. Journal of Physics: Condensed Matter 2017;29(27):273002. http://stacks.iop. $\operatorname{org} / 0953-8984 / 29 / \mathrm{i}=27 / \mathrm{a}=273002$.

[7] Lu T, Chen F. Multiwfn: A multifunctional wavefunction analyzer. Journal of Computational Chemistry 2012;33(5):580592. https://onlinelibrary.wiley.com/doi/abs/10.1002/jcc.22885.

[8] McGibbon RT, Beauchamp KA, Harrigan MP, Klein C, Swails JM, Hernández CX, et al. MDTraj: A Modern Open Library for the Analysis of Molecular Dynamics Trajectories. Biophysical Journal 2015;109(8):1528 - 1532.

[9] Michaud-Agrawal N, Denning EJ, Woolf TB, Beckstein O. MDAnalysis: A toolkit for the analysis of molecular dynamics simulations. J Comput Chem 2011;32(10):2319-2327.

[10] Richard J Gowers, Max Linke, Jonathan Barnoud, Tyler J E Reddy, Manuel N Melo, Sean L Seyler, et al. MDAnalysis: A Python Package for the Rapid Analysis of Molecular Dynamics Simulations. In: Sebastian Benthall, Scott Rostrup, editors. Proceedings of the 15th Python in Science Conference; 2016. p. 98 - 105.

[11] Frisch MJ, Trucks GW, Schlegel HB, Scuseria GE, Robb MA, Cheeseman JR, et al., Gaussian 16 Revision C.01; 2016. Gaussian Inc. Wallingford CT.

[12] Schaftenaar G, Vlieg E, Vriend G. Molden 2.0: quantum chemistry meets proteins. Journal of Computer-Aided Molecular Design 2017;31(9):789-800. https://doi.org/10.1007/s10822-017-0042-5.

[13] Werner HJ, Knowles PJ, Knizia G, Manby FR, Schütz M. Molpro: a general-purpose quantum chemistry program package. WIREs Comput Mol Sci 2012;2:242-253.

[14] To learn about QCSchema, please refer to its website;. https://molssi-qc-schema.readthedocs.io/en/latest/index.html.

[15] Barca GMJ, Bertoni C, Carrington L, Datta D, De Silva N, Deustua JE, et al. Recent developments in the general atomic and molecular electronic structure system. The Journal of Chemical Physics 2020;152(15):154102. https://doi.org/10. $1063 / 5.0005188$.

[16] Neese F. Software update: the ORCA program system, version 4.0. WIREs Computational Molecular Science 2018;8(1):e1327.

[17] Shao Y, Gan Z, Epifanovsky E, Gilbert ATB, Wormit M, Kussmann J, et al. Advances in molecular quantum chemistry contained in the Q-Chem 4 program package. Mol Phys 2015;113:184-215.

[18] Kühne TD, lannuzzi M, Del Ben M, Rybkin VV, Seewald P, Stein F, et al. CP2K: An electronic structure and molecular dynamics software package - Quickstep: Efficient and accurate electronic structure calculations. The Journal of Chemical Physics 2020;152(19):194103. https://doi.org/10.1063/5.0007045. 
[19] Parrish RM, Burns LA, Smith DGA, Simmonett AC, DePrince AE, Hohenstein EG, et al. Psi4 1.1: An Open-Source Electronic Structure Program Emphasizing Automation, Advanced Libraries, and Interoperability. Journal of Chemical Theory and Computation 2017;13(7):3185-3197. https://doi.org/10.1021/acs.jctc.7b00174.

[20] TURBOMOLE V7.4 2019, a development of University of Karlsruhe and Forschungszentrum Karlsruhe GmbH, 19892007, TURBOMOLE GmbH, since 2007; available from

http://www.turbomole.com.;.

[21] Smith DGA, Altarawy D, Burns LA, Welborn M, Naden LN, Ward L, et al. The MolSSI QCArchive project: An opensource platform to compute, organize, and share quantum chemistry data. WIREs Computational Molecular Science;n/a(n/a):e1491. https://onlinelibrary.wiley.com/doi/abs/10.1002/wcms.1491.

\section{GRAPHICAL ABSTRACT}

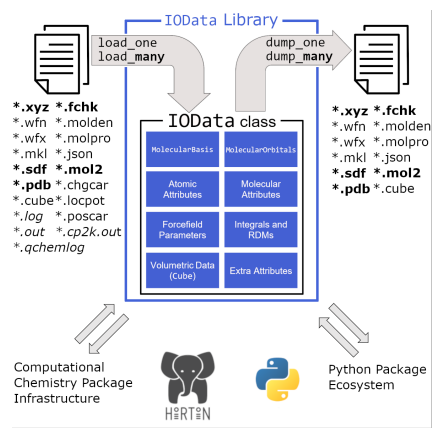

